\title{
Cognitive radio technology for flexible spectrum sharing
}

\begin{abstract}
Recent study by FCC Spectrum-Policy Task Force (SPTF) found that while the available spectrum becomes increasingly scarce, the assigned spectrum is significantly underutilized. Cognitive radio (CR) technology refers to a new type of radio hat uses real-time interaction with its environment to determine transmitter parameters such as frequency, power, and modulation. This paper presents a preliminary survey of CR with the goal of quantifying spectral occupancy and thereby gaining some insight into its feasibility. Two case studies were investigated: ultra-wide bandwidth technology as a transmission technique suitable for implementing a cognitive radio system was discussed, and a comparable measurement of TV spectrum band in an urban environment is also presented.
\end{abstract}

Keyword: Cognitive radio; FCC; Spectrum sensing; SPTF; TV; UWB 